\title{
Anti-PD1/ICOS Bispecific Monoclonal Antibody XmAb23104
}

National Cancer Institute

\section{Source}

National Cancer Institute. Anti-PD1/ICOS Bispecific Monoclonal Antibody XmAb23104.

NCI Thesaurus. Code C157578.

A humanized, Fc-engineered bispecific monoclonal antibody directed ag ainst both the human negative immunoregulatory checkpoint receptor, programmed cell death protein 1 (PD-1; PCD-1; CD279), and inducible T-cell co-stimulator (ICOS; CD278), with potential immunomodulating and antineoplastic activities. Upon administration, anti-PD1/ICOS bispecific monoclonal antibody XmAb23104 targets and binds to both PD-1 and ICOS expressed on certain T-cells, including tumor-infiltrating lymphocytes (TILs). This prevents the activation of PD-1 by its ligands, programmed cell death-1 lig and 1 (PD-L1) and PD-1 lig and 2 (PD-L2), and stimulates ICOS-mediated signaling, which promotes the activation of T-cells and enhances T-cell-mediated immune responses ag ainst tumor cells. Combined PD-1 blockade and ICOS stimulation may enhance T-cell activation and proliferation more than targeting each receptor individually. The eng ineered Fc domain increases the stability and half-life of the antibody. 